\title{
DOUBLE BINARY FORMS WITH THE CLOSURE PROPERTY*
}

\author{
BY \\ ARTHUR B. COBLE

\section{INTRODUCTION}

The polygons of Poncelet-a variable polygon whose $n$ vertices run over a fixed conic $C$ while its $n$ sides touch a fixed conic $K$-have long excited the interest of geometers. $\dagger$ If $t$ is the parameter of a variable point of $C$, $\tau$ the parameter of a variable tangent of $K$, the incidence condition of point $t$ of $C$ and line $\tau$ of $K$ is a double $(2,2)$ binary form

$$
(\alpha t)^{2}(a \tau)^{2}=0
$$

since each point $t$ of $C$ is on two lines $\tau$ of $K$ and each line $\tau$ of $K$ is on two points $t$ of $C$. When the two conics $C, K$ are so situated with respect to each other that the variable Poncelet polygons can be drawn, the double form (1) has the closure property. For a given vertex $t_{1}$ of a given polygon $P_{n}$ of $n$ sides determines in (1) the two sides $\tau_{1}, \tau_{n}$ on $t_{1}$. On each of these sides there is a vertex $t_{2}, t_{n}$ respectively in addition to $t_{1}$, the additional vertices being determined from (1). From these vertices additional sides $\tau_{2}, \tau_{n-1}$ are obtained, etc., until finally the polygon closes in to form a configuration $\Delta_{n, n}^{\mathbf{2}, 2}$ with parameters $t_{1}, \cdots, t_{n} ; \tau_{1}, \cdots, \tau_{n}$ of such sort that in (1) each of the $n t$ 's determines two of the $n \tau$ 's and vice versa. We shall say then that the form (1) admits the configuration $\Delta_{n, n}^{2,2}$. If the form admits $\infty^{1}$ such configurations, i. e., if it has the closure property for any initial $t_{1}$ (or $\tau_{1}$ ) then it is said to be poristic. A beautiful theorem $\ddagger$ of Poncelet states that the existence of one polygon $P_{n}$ implies the existence of $\infty^{1}$ polygons. Thus if the form (1) admits one $\Delta_{n, n}^{2,2}$ it admits infinitely many and is poristic.

A new departure in this field is due to $\mathrm{H}$. S. White $\S$ who showed that there exist $(3,3)$ double forms

$$
(\alpha t)^{3}(a \tau)^{3}
$$

* Presented to the Society, December 29, 1925; received by editors before December, 1925.

† Cf. G. Loria, I polygonì dĩ Poncelet, Turin, 1889; H. S. White, Poncelet polygons, S c i e n c e, Feb. 4, 1916, pp. 149-58.

$\ddagger$ Poncelet, Propriétés Projectives des Figures, 1822, p. 565 ff.

$\S$ Proceedings of the National Academy of Sciences, vol. 1 (1915), p. 464; vol. 2 (1916), p. 337. 
with configurations $\Delta_{7,7}^{3,3}$ Here again the existence of one configuration implies the existence of $\infty^{1}$ configurations. If, in space, (2) is the incidence condition of point $t$ of a twisted cubic $C^{3}$ with plane $\tau$ of a twisted cubic $K^{3}$ the configuration $\Delta_{7,7}^{3,3}$ consists of 7 points of $C^{3}$ and 7 planes of $K^{3}$ such that each of the 7 points is on three of the 7 planes and vice versa. It is however necessary in this case to specify the arrangement of incidences in the configuration. This may be done conveniently by giving the seven triads of points $t_{1}, \cdots, t_{7}$ (or merely their subscripts $1, \cdots, 7$ ) cut out by the seven planes $\tau_{1}, \cdots, \tau_{7}$ as in the scheme

$$
127.136 .145 .235 .246 .347 .567 \text {. }
$$

A few years later the writer* discussed the conditions for closure of the general double $(k, \kappa)$ binary form

$$
F=(\alpha t)^{k}(a \tau)^{\kappa}=0 .
$$

If there exists a set of $n$ values $t_{1}, \cdots, t_{n}$ of $t$ and a set of $\nu$ values $\tau_{1}, \cdots, \tau_{\text {, }}$ of $\tau$ such that each of the $n t$ 's determines in (3) $\kappa$ of the $\nu \tau$ 's and each of the $\nu \tau$ 's determines in (3) $k$ of the $n t$ 's where

$$
n \kappa=\nu k,
$$

then $F$ is said to admit the configuration $\Delta_{n, \nu}^{k, \kappa}$. If $F$ admits $\infty^{1}$ such configurations the double form has the closure property and is poristic. For such poristic forms certain fundamental theorems were derived. Thus the sets of $n$ values $t$ lie in an involution $(\gamma t)^{n}+\lambda(\delta t)^{n}=0$; the sets of $\nu$ values $\tau$ lie in an involution $(c \tau)^{\nu}+\lambda(d \tau)^{\nu}=0$ whose sets are projectively related to the sets of the first involution. Hence the determinant

$$
D_{n, \nu} \equiv\left|\begin{array}{ll}
(\gamma t)^{n} & (\delta t)^{n} \\
(c \tau)^{\nu} & (d \tau)^{\nu}
\end{array}\right|=F \cdot F^{\prime}
$$

factors into the form $F$ and a complementary form

$$
F^{\prime}=(\beta t)^{n-k}(b \tau)^{\nu-\kappa}
$$

such that the form $F^{\prime}$ admits the configurations $\Delta_{n, \nu}^{n-k, v-\kappa}$ complementary to $\Delta_{n, \nu}^{k, \kappa}$ i. e., $t$ in (6) determines the $\nu-\kappa$ values $\tau$ which are not determined in (3) by $t$. Conversely the determinant, $D_{n, \nu}=0$, is obviously poristic with configurations $\Delta_{n, \nu}^{n, \nu}$ and, if factorable, its factors are necessarily poristic. Particularly notable is the theorem $\dagger$

*A. B. Coble, Multiple binary forms with the closure property, A merican Journal of Mathematics, vol. 43 (1921), pp. 1-19.

† A. B. Coble, loc. cit., p. 3. 
(7) The necessary and sufficient conditions that a form $F_{k, \kappa}$ shall be poristic with configurations $\Delta_{n, \nu}^{k, \kappa}(n, \nu, k, \kappa$ integers subject to (4)) are (A) that the given form shall admit one such configuration and (B) that there shall exist a form $F_{n-k, v-\kappa}$ which shall admit the complementary configuration $\Delta_{n, \nu}^{n-k, k}$.

In addition to such theorems certain general methods of procedure are explained whose efficacy is shown by the derivation of twelve new types of poristic forms with configurations

$$
\begin{aligned}
& \Delta_{n, n}^{n-2, n-2}, \quad \Delta_{k, \kappa}^{k, k}, \quad \Delta_{k+1, k+1}^{k, k}, \quad \Delta_{2 n, n 2}^{2, n}, \quad \Delta_{2 n, n(n-1)}^{2, n-1}, \Delta_{2 n, n 2}^{2(n-1), 2(n-1)}, \\
& \begin{array}{rrrr}
2(n-1),(n-1)^{2} \\
2 n, n(n-1)
\end{array}, \quad \Delta_{7,7}^{4,4}, \quad \Delta_{4,8}^{2,3}, \quad \Delta_{5,10}^{2,4}, \quad \Delta_{5,10}^{8,1}, \quad \Delta_{6,8}^{8,4} \text {, }
\end{aligned}
$$

to which should be added the Poncelet type $\Delta_{n, n}^{2,2}$ and White's type $\Delta_{7,7}^{3,3}$ Six of these new types embody an infinite number of cases for arbitrary choice of the integer $n$. One type embodies a doubly infinite number of cases for arbitrary choice of $k, \kappa$. This type however is the obvious poristic determinant $D_{k, k}$ in (5). The other types are particular cases selected because they were manageable.

Quite recently* Louise D. Cummings exhibited two poristic forms $F_{3,8}$ each with configurations $\Delta_{6,6}^{3,3}$ which she calls "double sextettes," the grouping for the two forms being essentially different. Professor Cummings' method is novel. She examines first the conditions on the coefficients of the form $F_{3,3}$ that it may admit one double sextette $\Delta_{6,6}^{3,3}$ of the prescribed kind. The additional integral conditions on the coefficients of the form that it may admit a consecutive sextette are then determined. If both types of conditions can be satisfied the resulting form is necessarily poristic. $\dagger$

It occurred to the present author to examine these two porisms by the methods of his earlier paper. $\ddagger$ This discussion is carried out in $\S 2$ and $\S 4$ after a preliminary discussion in $\S 1$ of the associated group problem. A striking result of the geometric argument employed is that Miss Cummings'

* Louise D. Cummings, Proceedings International Mathematical Congress, Toronto (1924), not yet published; Bulletin of the American Mathematical Society, vol. 31 (1925), pp. 266-74.

† These remarks are based on the exposition contained in the $\mathrm{B} \mathrm{ull}$ e $\mathrm{t}$ in paper. I have seen the manuscript of the Toronto paper but recall no details.

$\ddagger$ Loc. cit. 
poristic $(3,3)$ form is factorable* in each of the two cases-a fact not recognized by Miss Cummings and indeed not easily recognizable in the explicitly given form $F$. $\dagger$ Moreover, the character of the factors is so clearly defined that each case appears as an individual in a doubly infinite series. One of these series may properly be called the "Poncelet polygons of $n$ sides in space of $r$ dimensions." The other series has a characteristic grouping which, for reasons explained later, may be called "cyclically imprimitive." For each series the explicit algebraic construction of the poristic forms is given.

In $\S 6$ all poristic forms $F_{3,3}$ with configurations $\Delta_{n, n}^{3,3}(n<10)$ are determined. Of these all but two, the double sextettes of White, and a novel case of double octettes, are included in the general classes mentioned above. Except for these two and one other, all the poristic $F_{3,3}$ 's $(n<10)$ are factorable.

\section{THE GROUP PROBLEM FOR PORISTIC FORMS}

It has been pointed out $\ddagger$ that a configuration $\Delta_{n, \nu}^{k, \kappa}$ is not defined until the $n t$ 's have been arranged in $\nu$ sets of $k$ in such a way that each $t$ is included in $\kappa$ of the $\nu$ sets. A convenient way of indicating such an arrangement is to write down the $k$-ads formed from the $t$ 's and to assign to them in some order the values of the $\nu \tau$ 's. Such an arrangement is given in the introduction for White's porism. However, not every such arrangement can lead to a porism. An additional condition is given in the following theorem :

(9) For a poristic form $F_{k, \kappa}$ with configurations $\Delta_{n, \nu}^{k, \kappa}$ the arrangement of $n t$ 's into $\nu$ sets of $k$ each must be such as will admit the permutations of a group transitive on the $n$ t's.

For if the form $F_{k, \kappa}(k \leqq \kappa)$ is interpreted, as in the author's earlier paper, $\S$ as the incidence condition of point, $t$, of a rational norm curve $N^{k}$ in $S_{k}$ with $S_{k-1}, \tau$, of a rational curve $R^{\kappa}$ in $S_{k}$, the points $t$ of the $\infty^{1}$ configurations run over $N^{k}$ and a configuration may be drawn starting with any one point $t$ of a configuration in precisely the same way as by starting with any other point $t^{\prime}$ of the same configuration. Thus if $t$ be replaced by $t^{\prime}$ it must be possible to replace any other $t$, say $t_{1}$, by some $t$, say $t_{1}^{\prime}$, in such a way that the configuration, i. e., the distribution of $n t$ 's into $k$-ads, is unaltered.

\footnotetext{
* I have a vague recollection of examining the configurations in connection with the preparation of my earlier paper (loc. cit.) and discarding them for some reason-probably this very factorization. A valuable feature of Miss Cummings' contribution is that examples are given of poristic forms which have considerable geometric interest in spite of their rather simple algebraic character.

† Bulletin of the American Mathematical Society, loc. cit., p. 268.

$\ddagger$ A. B. Coble, loc. cit., p. 6.

§ Loc. cit., p. 7.
} 
If we examine possible arrangements for the double sextettes $\Delta_{6,6}^{3,3}$ we find four cases:

123. 234. 345. 456. 561. 612 ;

142. 145. 253. 256. 361. 364 ;

123. 124. 125. 456. 536. 346 ;

123. 124. 135. 256. 346. 456 .

We see that in $(\gamma)$ the pair 12 occurs in three triads, the pairs $36,46,56$ occur in two triads, and other pairs occur in one or no triads. Thus the group of $(\gamma)$ is intransitive with $12,345,6$ as systems of transitivity. Similarly in the triads of $(\delta)$ the pairs $12,13,46,56$ each occur twice and all other pairs occur once except 16 which does not occur at all. Thus the group of $(\delta)$ is intransitive with 16,2345 as systems of transitivity. On the other hand $(\alpha)$ is invariant under a transitive dihedral $g_{2.6}$ and $(\beta)$ is invariant under a transitive $g_{24}$. Both groups contain a cyclic element of period six as is noted by Miss Cummings. For $(\alpha)$ the cyclic $g_{6}$ is invariant under $g_{2.6}$; for $(\beta)$, however, the cyclic $g_{6}$ is one of a number conjugate under $g_{24}$. Thus the emphasis is to be laid on the transitivity rather than the cyclic character of the group. It is, however, quite proper to call the configuration $(\alpha)$ cyclic or polygonal. We may better call the configuration $(\beta)$ cyclically imprimitive. For in $(\beta)$ the six $t$ 's divide into three cyclic pairs $14,25,36$ and the triads are formed by combining a pair with a member of the next pair. For both $(\alpha)$ and $(\beta)$ the groups are imprimitive with $14,25,36$ as systems of imprimitivity.

An obvious further condition on the $\nu k$-ads is that they shall not contain a set of $\nu_{1}<\nu k$-ads such that in the $\nu_{1} k$-ads alone each $t$ of a set of $n_{1}<n t$ 's is included in $\kappa$ of the $\nu_{1}$ sets. In such case the given configuration would be merely an aggregate of smaller configurations.

In the next paragraph the case $(\beta)$ is examined and the algebraic nature of its poristic form is determined.

2. THE PORISTIC FORM FOR THE DOUBLE SEXTETTE $(\beta)$

We will assume the existence of a poristic form $F_{3,3}$ with $\infty^{1}$ configurations $\Delta_{6,6}^{3,3}$ determined by the arrangement

$$
\text { 142. 145. 253.256.361. } 364 \text {, }
$$

and will call the triads of six $t^{\prime}$ s in the order given $\tau_{2}, \tau_{5}, \tau_{3}, \tau_{6}, \tau_{1}, \tau_{4}$ respectively. Let then $G_{6,6}$ be that rational covariant of $F_{3,3}$ which expresses that $t, t^{\prime}$ determine in $F_{3,3}=0$ a common $\tau$. This form $G_{6,6}$, symmetric in $t, t^{\prime}$, 
coördinates to $t=t_{1}$ the values $t^{\prime}=t_{2}, t_{5}, t_{3}, t_{6}$ and $t^{\prime}=t_{4}$ taken twice. Hence $G_{6,6}$ is rationally factorable,

$$
G_{6,6}=\left(I_{1,1}\right)^{2} \cdot K_{4,4},
$$

where $\bar{I}_{1,1}$ is a symmetric linear form in $t, t^{\prime}$, and $K_{4,4}$ a symmetric quartic form in $t$ and in $t^{\prime}$. Thus $I_{1,1}$ is an ordinary involution of pairs of points and $t_{1}, t_{4} ; t_{2}, t_{5} ; t_{3}, t_{6}$ are pairs of this involution. Similarly if $\bar{G}_{6,6}$ is the rational covariant of $F_{3,3}$ which expresses that $\tau, \tau^{\prime}$ determine in $F=0$ a common $t$, then $\bar{G}_{6,6}$ factors, i. e.,

$$
\bar{G}_{6,6}=\left(\bar{I}_{1,1}\right)^{2} \cdot \bar{K}_{4,4}
$$

where $\bar{I}_{1,1}$ is an involution with pairs $\tau_{1}, \tau_{4} ; \tau_{2}, \tau_{5} ; \tau_{3} \tau_{6}$.

The involutions $I_{1,1}$ and $\bar{I}_{1,1}$ can be obtained rationally from the forms $G$ and therefore also from $F_{3,3}$, and on multiplying them respectively by $t-t^{\prime}, \tau-\tau^{\prime}$ the pairs of the involutions are obtained. We observe at once that the three $\tau$ 's determined by a given $t$ in $F_{3,3}=0$ divide into a pair of the involution $\bar{I}_{1,1}$ and an isolated $\tau$; thus each $t$ determines one $\tau$ and conversely each $\tau$ is thereby determined by one $t$. Hence the six $t$ 's and six $\tau$ 's are projective and $F_{3,3}$ factors into

$$
F_{3,3}=F_{1,1} \cdot F_{2,2},
$$

where $F_{1,1}$ determines the projectivity between $t$ and $\tau$. For example $t_{1}$ determines in $F_{3,3}=0$ the pair $\tau_{2}, \tau_{5}$ of $\bar{I}_{1,1}$ and also $\tau_{1}$; whence $F_{1,1}=0$ is a projectivity between $t_{i}, \tau_{i}(i=1, \cdots, 6)$.

The factors of $F_{3,3}$ are gotten by rational processes as follows. The resultant of $F_{3,3}$ and $\bar{I}_{1,1}$ in $\tau$ is of degree 3 in $t$ and 3 in $\tau^{\prime}$ and on replacing $\tau^{\prime}$ by $\tau$ this resultant coördinates to $t_{1}$ the values $\tau=\tau_{2}, \tau_{5}, \tau_{4}$, whereas $F_{3,3}$ itself coördinates to $t_{1}$ the values $\tau=\tau_{2}, \tau_{5}, \tau_{1}$. Thus $F_{2,2}$ is the common factor of $F_{3,3}$ and this resultant, and $F_{1,1}$ is the residual factor of $F_{3,3}$.

For a canonical form we naturally take the projectivity $F_{1,1}=0$ to be the identical projectivity $t-\tau=0$ and thus regard $t, \tau$ as superposed ranges. If we take $0, \infty$ to be the double points of $I_{1,1}$ and therefore also of $\bar{I}_{1,1}$ the pairs are respectively $t,-t$ and $\tau,-\tau$. Moreover, the factor $F_{2,2}=0$ coördinates either to $t$ or its partner $-t$ the same pair $\tau,-\tau$, whence $F_{2,2}=0$ has the form

$$
a \tau^{2} t^{2}+b \tau^{2}+c t^{2}+d=0 .
$$

Thus the form is imprimitive* and arises from $a \sigma s+b \sigma+c s+d=0$ by the substitutions $\sigma=\tau^{2}, s=t^{2}$. This projectivity between $\sigma$ and $s$ (when each

* A. B. Coble, loc. cit., p. 6. 
is on the same scale) is periodic of period three. For $t,-t=t_{1}, t_{4}$ determine, in $F_{2,2}=0, \tau,-\tau=\tau_{2}, \tau_{5}$ which in the projectivity $\tau=t$ (or $s=\sigma$ ) are $t,-t=$ $t_{2}, t_{5}$. Similarly we pass from the pair $t_{2}, t_{5}$ to the pair $t_{3}, t_{6}$ and again to the original pair $t_{1}, t_{4}$. If the fixed points of the projectivity between $s$ and $\sigma$ are taken to be $1, f$ then the final canonical form of the required poristic form $F_{3,3}$ is

$$
F_{3,3}=(t-\tau)\left\{\frac{t^{2}-f}{t^{2}-1}-\epsilon \frac{\tau^{2}-f}{\tau^{2}-1}\right\} \quad\left(\epsilon=e^{2 \pi i / 3}\right) .
$$

Thus $F_{3,3}$ has one absolute constant.

We observe that each factor of $F_{3,3}$ is poristic in a rather trivial way. Thus $t-\tau$ admits configurations $\Delta_{1,1}^{1,1}$ and the residual factor admits configurations $\Delta_{2,2}^{2,2}$ of imprimitive type. The effect of taking the product is to distribute the $\Delta_{2,2}^{2,2}$ into closed systems of three which form the double sextettes.

Miss Cummings gives the following as a normal form for this case :

$$
\begin{aligned}
& F(x, y)=12.35 .56 \text {. ae.cf. } x b . x c . x d \text {. } y 1 . y 2 . y 4 \\
& +61.25 .14 \text {. bf.ce. } x a . x c . x d . y 2 . y 3 . y 5 \quad(x a=x-a) \\
& +12.15 .25 \text {. ce.cf . } x a . x b . x d \text {. y3.y4.y6 } \quad\left(12=t_{1}-t_{2}\right) \\
& +15.23 .26 \text {. cf.de. } x a . x b . x c . y 4 . y 5 . y 1=0
\end{aligned}
$$

where $a, b, c, d, e, f$ correspond to $\tau_{2}, \tau_{3}, \tau_{4}, \tau_{5}, \tau_{6}, \tau_{1}$ above. She finds as the condition for a single $\Delta_{6,6}^{3,3}$ the projectivity of the six $t^{\prime}$ 's and six $\tau$ 's and as the additional condition for a porism the fact that $t_{1}, t_{4} ; t_{2}, t_{5} ; t_{3}, t_{6}$ are pairs of an involution. If then in $F(x, y)$ the six $t$ 's and six $\tau$ 's in order are replaced by $\lambda, \mu, \nu,-\lambda,-\mu,-\nu$ the form $F(x, y)$ becomes, after a rather awkward reduction,

$$
\begin{aligned}
& 4 \lambda \mu(x-y)\left[\left(\lambda^{4}+\mu^{4}+\nu^{4}-\mu^{2} \nu^{2}-\nu^{2} \lambda^{2}-\lambda^{2} \mu^{2}\right) x^{2} y^{2}\right. \\
& \quad+\left(3 \lambda^{2} \mu^{2} \nu^{2}-\lambda^{4} \nu^{2}-\mu^{4} \lambda^{2}-\nu^{4} \mu^{2}\right) x^{2}+\left(3 \lambda^{2} \mu^{2} \nu^{2}-\lambda^{4} \mu^{2}-\mu^{4} \nu^{2}-\nu^{4} \lambda^{2}\right) y^{2} \\
& \left.\quad+\left(\mu^{4} \nu^{4}+\nu^{4} \lambda^{4}+\lambda^{4} \mu^{4}-\left\{\lambda^{2}+\mu^{2}+\nu^{2}\right\} \lambda^{2} \mu^{2} \nu^{2}\right)\right] .
\end{aligned}
$$

The second factor is satisfied by $x^{2}, y^{2}=\lambda^{2}, \nu^{2}$ and therefore, due to the invariance of the coefficients under cyclic permutation of $\lambda^{2}, \mu^{2}, \nu^{2}$, is satisfied also by $x^{2}, y^{2}=\mu^{2}, \lambda^{2}$ and $\nu^{2}, \mu^{2}$, whence the period 3 and normal form (10) of the second factor is confirmed. 
3. THE PORISTIC FORM $F_{r+1, r+1}$ WITH CYCLICALLY IMPRIMITIVE CONFIGURATIONS $\Delta_{r s, r s}^{r+1, r+1}$ AND RELATED PORISTIC FORMS

Consider an array of $r s$ values of $t$ arranged in matrix form with $s$ rows of $r$ elements each as in

$$
\begin{aligned}
& t_{1,1} t_{1,2} \cdot \cdot \cdot t_{1, r} \\
& t_{2,1} t_{2,2} \cdot \cdot \cdot t_{2, r} \\
& \cdot \cdot \cdot \cdot \cdot \cdot \cdot \cdot \\
& t_{s, 1} t_{s, 2} \cdot \cdot \cdot t_{s, r}
\end{aligned}
$$

We consider the $r s$ sets of $r+1 t$ 's obtained by adjoining to the set of $r t$ 's in one row any one $t$ of the next lower row and to the set of $r t$ 's in the last row any one $t$ of the first row. The typical set is then

$$
\tau_{i+1, j}: \quad t_{i, 1}, t_{i, 2}, \cdot \cdot t_{i, r}, t_{i+1, j} \quad\left(\begin{array}{l}
i=1, \cdot \cdot, s ; s+1=1 \\
j=1, \cdot \cdot, r
\end{array}\right) .
$$

We assume the existence of a poristic form $F_{r+1, r+1}$ with closed sets of $r s$ values of $t$ and $r s$ values of $\tau$ such that the values of $\tau$ determine sets of $r+1 t$ 's as in the arrangement indicated. The set of $\tau$ 's thereby associated to a given $t$ is

$$
t_{i+1, j}: \quad \tau_{i+2,1}, \tau_{i+2,2}, \cdot \cdot \cdot \tau_{i+2, r}, \tau_{i+1, j} .
$$

The form $G_{r(r+1), r(r+1)}$ symmetric in $t$ and $t^{\prime}$, which expresses that $t$ and $t^{\prime}$ determine in $F_{r+1, r+1}$ a common $\tau$, coördinates to $t=t_{i, j}$ the values $t^{\prime}$ found in the row $t_{i-1}$, the row $t_{i+1}$, and also the values in the row $t_{i}$ other than $t_{i, j}$ each taken $r$ times. This $r$-fold factor can be isolated rationally and gives rise to a form $H_{r-1, r-1}$ symmetric in $t, t^{\prime}$ which for any $t$ determines the remaining $t$ 's in the same row. Thus the sets of $r t$ 's in a row of the original array lie in an involution and $H_{r-1, r-1}=0$ is the condition that $t, t^{\prime}$ belong to the same set of the involution. By a similar argument the $\tau_{i, j}$ 's in a row (i. e. with the same $i$ ) are sets of an involution which is determined by a form $\bar{H}_{r-1, r-1}=0$ symmetric in $\tau, \tau^{\prime}$.

We consider then the resultant of $F_{r+1, r+1}$ and $\bar{H}_{r-1, r-1}$ in $\tau$, a form of order $r^{2}-1$ in $t$ and in $\tau^{\prime}$ (which we henceforth call $\tau$ ). This resultant $R$ coördinates to $t=t_{i, j}$ the $\tau^{\prime}$ 's of the row $\tau_{i+1}$ each taken $r-1$ times and the $\tau$ 's of the row $\tau_{i}$ except $\tau_{i, j}$. Since $F_{r+1, r+1}$ coördinates to $t=t_{i, j}$ the $\tau$ 's of the row $\tau_{i+1}$ and $\tau_{i, j}$ there follows that $R$ and $F_{r+1, r+1}$ have a rational common factor $H_{r, r}$ which coördinates to $t=t_{i, j}$ the $\tau$ 's of the row $\tau_{i+1}$ and $F_{r+1, r+1}$ has a residual factor $L_{1,1}$ which coördinates to $t=t_{i, j}$ the value $\tau=\tau_{i, j}$. Hence the sets of $r s t$ 's and $r s \tau$ 's are projective under a projectivity (fixed 
for the porism) which sends $t_{i, j}$ into $\tau_{i, j}$. If we take the parameter $\tau$ in such fashion that the projectivity is given by $t-\tau=0$ then $t_{i, j}=\tau_{i, j}$. Thus $F$ has the factor $t-\tau$ and the residual factor $H_{r, r}$ is imprimitive, i. e., any $t$ of a row determines the next row of $\tau$ 's, now the same as the next row of $t$ 's. If then we write the involutions in which these sets lie as $(\gamma t)^{r}-l(\delta t)^{r}=0$, $(\gamma \tau)^{r}-\lambda(\delta \tau)^{r}=0$, the form $H_{r, r}$ becomes bilinear in $l, \lambda$. Moreover, since $H_{r, r}$ coördinates one row to the next this bilinear relation is cyclic and of period $s$ and can be written as

$$
\frac{l-f_{1}}{l-f_{2}}-\epsilon \frac{\lambda-f_{1}}{\lambda-f_{2}}=0,
$$

where $\epsilon$ is a primitive sth root of unity. Hence $F_{r+1, r+1}$ has the canonical form

$$
(t-\tau)\left\{\frac{(\gamma t)^{r}-f_{1}(\delta t)^{r}}{(\gamma t)^{r}-f_{2}(\delta t)^{r}}-\epsilon \frac{(\gamma t)^{r}-f_{1}(\delta t)^{r}}{(\gamma t)^{r}-f_{2}(\delta t)^{r}}\right\}=0 .
$$

Conversely a form of this type is poristic with configurations of the type described. For if on a line $\xi$ with coördinate $\lambda, l$ on the same scale we indicate the $s$-ads of a cyclic collineation and then put the line $\xi$ into $(1, r)$ correspondence with a line $\eta$ with $t, \tau$ on the same scale, successive points of the original cyclic sets of $s$ become successive rows of the original array. The addition of the factor $t-\tau$ then destroys the imprimitivity and opens a passage from the two successive sets to adjacent sets.

The involution $(\gamma t)^{r}-l(\delta t)^{r}=0$ has $2 r-5$ absolute constants and these with $f_{1}, f_{2}$ give rise to $2 r-3$ absolute constants in the poristic form. Thus we have proved that

(12) For all values of $r \geqq 2$ and $s \geqq 2$ there exist poristic forms $F_{r+1, r+1}$ with configurations $\Delta_{r s, r s}^{r+1, r+1}$ of the cyclically imprimitive type described above. These forms contain $2 r-3$ absolute constants.

The case $r=2$ with a form $F_{3,3}$ and configurations $\Delta_{2 s, 2 s}^{3,3}$ in ordinary space merits some consideration. The $s$ pairs of points $t$ lie on $s$ bisecants of a cubic curve $C^{3}$ and the $s$ bisecants are generators of one system of a quadric $Q$ which contains $C^{3}$ since the $s$ pairs are in an involution. A plane $\tau$ contains one pair and is therefore on a generator and is a tangent plane of $Q$. Thus the quadric $Q$ as an envelope contains the planes of the cubic curve $K^{3}$. If $\lambda, \mu$ are the parameters of the two sets of generators such that the generators $\lambda, \mu$ meet in a point $x$ of $Q$ and lie in a plane $\xi$ of $Q$, the parametric equations of $Q$ as a point locus or locus of planes are

$$
\begin{array}{llll}
x_{0}=\lambda \mu, & x_{1}=\lambda, & x_{2}=\mu, & x_{3}=1 ; \\
\xi_{0}=1, & \xi_{1}=-\mu, & \xi_{2}=-\lambda, & \xi_{3}=\lambda \mu .
\end{array}
$$


If the generators $\lambda$ are bisecants of $C^{3}$ the equation of $C^{3}$ on $Q$ is given by the $(1,2)$ form $(a \lambda)(b \mu)^{2}=0$. Since the generators $\lambda$ cut out the pairs of points $t$ in the configuration $\Delta_{2 s, 2 s}^{3,3}$ the parameters $\lambda$ of the $s$ pairs are cycloprojective and may be taken as $\lambda, \epsilon \lambda, \epsilon^{2} \lambda, \cdots, \epsilon^{s-1} \lambda$. The parameter on $C^{3}$ may be taken to be $t=\mu$. If then we take a point $\mu$ on $C^{3}$ the corresponding plane of $K^{3}$ is the plane on the same point and the preceding bisecant $\epsilon^{8-1} \lambda$, a plane which cuts $Q$ in the generators $\epsilon^{s-1} \lambda, \mu$. If we solve for $\lambda$ in $(a \lambda)(b \mu)^{2}=0$ and obtain $\lambda=(q \mu)^{2} /(r \mu)^{2}$ then we have for point $x(\lambda, \mu)$ on $C^{3}$ and plane $\xi\left(\epsilon^{\boldsymbol{s}-1} \lambda, \mu\right)$ the coördinates

$$
\begin{array}{llll}
x_{0}=\mu(q \mu)^{2}, & x_{1}=(q \mu)^{2}, & x_{2}=\mu(r \mu)^{2}, & x_{3}=(r \mu)^{2} ; \\
\xi_{0}=\epsilon(r \mu)^{2}, & \xi_{1}=-\epsilon \mu(r \mu)^{2}, & \xi_{2}=-(q \mu)^{2}, & \xi_{3}=\mu(q \mu)^{2} .
\end{array}
$$

Then the incidence condition of plane $\xi\left(\mu^{\prime}\right)$ and point $x(\mu)$ is

$$
\left(\mu-\mu^{\prime}\right)\left[\epsilon \frac{(q \mu)^{2}}{(r \mu)^{2}}-\frac{\left(q \mu^{\prime}\right)^{2}}{\left(r \mu^{\prime}\right)^{2}}\right]
$$

which is of the same general form as (10). Hence

(13) Given a cubic curve $C^{3}$ on a quadric $Q$; if $C^{3}$ is transformed into a cubic curve $K^{3}$ by a correlation which is the product of a collineation of period $s$ which leaves every unisecant generator of $C^{3}$ unaltered and a polarity in $Q$, then $C^{3}$ and $K^{3}$ contain configurations $\Delta_{2 s, 2 s}^{3,3}$. The bisecant generators of $C^{3}$ constitute an infinity of lines bisecant to $C^{3}$ which are also axes of $K^{3}$.

Miss Cummings remarks that the double sextettes $(\beta)$ furnish an infinity of such bisecant-axes which are not of the usual Hurwitz type. We see above that the case of an infinity of bisecant-axes for $C^{3}, K^{3}$ may arise in an infinity (for variable $s$ ) of ways, all projectively distinct. This, however, is not remarkable since $C^{3}, K^{3}$ are on the same quadric with one set of generators as bisecant-axes.

We think of the $r s$ 's arranged in an array like the $t$ 's, each $\tau$ being equal to the like-placed $t$. Then the form $H_{r, r}$ which appears as a factor in (11) and which we denote now by $H_{r, r}(\epsilon)$ correlates to any $t$ all the $\tau$ 's in the next row. A similar form $H_{r, r}\left(\epsilon^{m}\right)$ correlates to any $t$ all the $\tau$ 's in the row $m$ times removed from the one in which $t$ occurs. In this way for $m=1, \cdots, s-1$ any row of $\tau$ 's is correlated to $t$ except the one in which it occurs. The form $H_{r-1, r-1}$ correlates to $t$ all the $\tau$ 's in its own row except $\tau=t$, and the form $(t-\tau)$ correlates to $t$ its own $\tau$. It is evident then that the products

$$
\begin{aligned}
& (t-\tau) . H_{r, r}\left(\epsilon^{a_{1}}\right) \ldots \ldots . H_{r, r}\left(\epsilon^{a_{j}}\right), \\
& H_{r-1, r-1} \cdot H_{r, r}\left(\epsilon^{a_{1}}\right) \ldots . . H_{r, r}\left(\epsilon^{a_{j}}\right),
\end{aligned}
$$


where $a_{1}, \cdots, a_{j}$ is a selection from the integers $1, \cdots, s-1$, are poristic and we see that

(16) There exist poristic forms $F_{j r+1, j r+1}$ with configurations $\Delta_{r s, r s}^{j r+1, j r+1}$; and poristic forms $F_{j r+r-1, j r+r-1}$ with configurations $\Delta_{r s, r s}^{j r+r-1, j r+r-1}$ all of the cyclically imprimitive type where $1 \leqq j \leqq s-1$.

In forming products like (14), (15) those with both factors $t-\tau, H_{r-1, r-1}$ are omitted since they are imprimitive, i. e., reducible to forms of lower degrees in $t, \tau$ by rational substitutions.

\section{The PORISTIC FORM FOR THE DOUBLE SEXTETTE $(\alpha)$}

Let $F_{3,3}$ be poristic with configurations $\Delta_{6,6}^{3,3}$ determined by the arrangement

$$
123.234 .345 .456 .561 .612
$$

whose triads in the order given are denoted by $\tau_{2}, \tau_{3}, \tau_{4}, \tau_{5}, \tau_{6}, \tau_{1}$. The configuration may be regarded as a skew-hexagon in space with six ordered vertices $t_{1}, \cdots, t_{6}$, six ordered sides $t_{1} t_{2}, \cdots, t_{5} t_{6}, t_{6} t_{1}$ each joining two consecutive vertices, and six ordered planes $\tau_{1}=612, \cdots, \tau_{5}=456, \tau_{6}=561$ each on three consecutive vertices. The form $F_{3,3}=0$ determines the incidence of points and planes of the hexagon.

If $t, t^{\prime}$ determine in $F_{3,3}=0$ the same $\tau$, i. e., if distinct vertices $t, t^{\prime}$ are on the same plane of the hexagon, they satisfy a symmetric form $G_{6,6}=0$ which coördinates to $t_{1}$ the values $t^{\prime}=t_{2}, t_{6}$ each twice and $t_{3}, t_{5}$ each once. Thus $G_{6,6}$ has two rational factors $P_{2,2}^{(1)}$ and $P_{2,2}^{(2)}$ each symmetric in $t, t^{\prime}$. The form $P_{2,2}^{(1)}$ is satisfied by adjacent vertices $t, t^{\prime}$; the form $P_{2,2}^{(2)}$ is satisfied by vertices $t, t^{\prime}$ twice removed from each other. If we let the two values $t^{\prime}$ determined by $t$ in $P_{2,2}^{(1)}$ be taken for $t$ in $P_{2,2}^{(2)}$, the four values $t^{\prime}$ then obtained include the two already known from $P_{2,2}^{(1)}$ and two which are the vertices thrice removed from $t$ and which are determined from a rationally known $P_{2,2}^{(3)}$ In the present case of a hexagon the vertices determined by $t=t_{1}$ in $P_{2,2}^{(3)}$ consist of $t_{4}$ taken twice, whence $P_{2,2}^{(3)}=\left(P_{1,1}\right)^{2}$. Thus opposite vertices of the $\infty^{1}$ hexagons are in a quadratic involution, $P_{1,1}=0$.

By the same argument there exist forms symmetric in $\tau, \tau^{\prime}: R_{2,2}^{(1)}, R_{2,2}^{(2)}, R_{1,1}$, which for given $\tau$ determine respectively the adjacent planes, the planes twice removed and the opposite plane, of the hexagon with respect to the plane $\tau$. Again the opposite planes of the $\infty^{1}$ hexagons are in a quadratic involution, $R_{1,1}=0$.

We observe that the three planes $\tau_{6}, \tau_{1}, \tau_{2}$ on the vertex $t_{1}$ form an unsymmetrical triad in that $\tau_{1}$ has a side in common with $\tau_{6}, \tau_{2}$ while $\tau_{6}, \tau_{2}$ 
have only the vertex $t_{1}$ in common. This indicates a factorization of the form $F_{3,3}$ into a product $Q_{1,1} \cdot Q_{2,2}$. The factors are rationally obtained as follows. The resultant in $t$ of $P_{2,2}^{(1)}$ and $F_{3,3}$, of degree 6 in $t^{\prime}$ and in $\tau$, coördinates to $t^{\prime}=t_{1}$ the values $\tau_{1}$ twice and $\tau_{2}, \tau_{3}, \tau_{5}, \tau_{6}$. Hence this resultant has a squared factor $Q_{1,1}$ and a residual quartic factor. Thus the factors $Q_{1,1}, Q_{2,2}$ of $F_{3,3}$ are rationally known. The factor $Q_{1,1}$ correlates $t_{i}$ to $\tau_{1}$ $(i=1, \cdots, 6)$; the factor $Q_{2,2}$ correlates $t_{1}, \cdots, t_{6}$ in order to the following pairs of $\tau$ 's : 62.13 .24 .35 .46 .51 .

We make a change of variable $\tau$ so that $Q_{1,1}$ becomes $t-\tau$, i. e., every $\tau_{i}$ is equal to $t_{i}$. We also choose the involution $P_{1,1}$ to be $t^{\prime}=-t$, whence $R_{1,1}$ is $\tau^{\prime}=-\tau$. Then the values of the six $t^{\prime}$ s and six $\tau^{\prime}$ s are

$$
t_{1}, t_{3}, t_{5}=\tau_{1}, \tau_{3}, \tau_{5}=\lambda, \mu, \nu ; \quad t_{4}, t_{6}, t_{2}=\tau_{4}, \tau_{6}, \tau_{2}=-\lambda,-\mu,-\nu .
$$

We observe that $Q_{2,2}$ correlates to $t_{1}, t_{3}, t_{5}$ the pairs formed from $\tau_{4}, \tau_{6}, \tau_{2}$ and to $t_{4}, t_{6}, t_{2}$ the pairs formed from $\tau_{1}, \tau_{3}, \tau_{5}$. Thus $Q_{2,2}$ admits two distinct configurations $\Delta_{3,3}^{2,2}$. If now we change the sign of $\tau$ then $Q_{2,2}$ is satisfied by any pair $t, \tau$ of $\lambda, \mu, \nu$ or any pair $t, \tau$ of $-\lambda,-\mu,-\nu$, i. e., $t, \tau$ are roots of a cubic of the pencil $\left(a_{0} t^{3}+a_{1} t^{2}+a_{2} t+a_{3}\right)+r\left(a_{0} t^{3}-a_{1} t^{2}+a_{2} t-a_{3}\right)$ and $F_{3,3}$ has the form

$$
(t+\tau)\left[\alpha t^{2} \tau^{2}+\beta\left(t^{2}+\tau^{2}\right)+\gamma t \tau+\delta\right] .
$$

We observe that the configurations $\Delta_{6,6}^{3,3}$ of (17) are formed by tying together two configurations $\Delta_{3,3}^{2,2}$ of the factor $Q_{2,2}$ by means of the other factor $Q_{1,1}$.

\section{Generalized Poncelet polygons}

We consider a configuration $\Delta_{n, n}^{r, r}(3 \leqq r \leqq n-3)^{*}$ of $n t^{\prime}$ 's, $t_{1}, \cdots, t_{n}$ and $n \tau$ 's, $\tau_{1}, \cdots, \tau_{n}$ such that each $\tau$ is coördinated to $r$ of the $t$ 's by the following scheme :

$$
123 \cdots r .234 \cdots r, r+1.34 \cdots r+1, r+2 \cdots \cdot n 12 \cdots r-1,
$$

which admits the cyclic group $(12 \cdots r \cdots n)$. This configuration will be called a "polygon in space $S_{r}$ of $r$ dimensions." We shall call $t_{1}, \cdots, t_{n}$ the points or vertices of the polygon; $12,23, \cdots, n 1$ the lines or sides of the polygon; $123,234, \cdots, n 12$ the planes of the polygon; and finally $12 \cdots r, \cdots, n 12 \cdots r-1$ the $S_{r-1}$ 's or faces of the polygon. Naturally such a polygon in $S_{r}$ is self dual and is determined by the cyclic order of its $n$ vertices $t$ or of its $n$ faces $\tau$.

We first assume the existence of a poristic form $F_{r, r}$ which admits $\infty^{1}$ such configurations, find the necessary conditions which this imposes on

\footnotetext{
* For the cases $r=2, n-2, n-1$, cf. A. B. Coble, loc. cit., p. $17,1^{\circ}, 2^{\circ}, 4^{\circ}$.
} 
$F_{r, r}$, and finally show how these conditions can be satisfied and the poristic form actually constructed.

If distinct $t, t^{\prime}$ determine in $F_{r, r}$ a common $\tau$, then $t, t^{\prime}$ satisfy a symmetric form $G$ of order $r^{2}-r$ in each. For given $t_{1}, G$ determines the vertices $t_{2}, t_{n}$ once removed from $t_{1}$ each $r-1$ times, the vertices $t_{3}, t_{n-1}$ twice removed from $t_{1}$ each $r-2$ times, etc., whence

$$
G=\left[P_{2,2}^{(1)}\right]^{r-1}\left[P_{2,2}^{(2)}\right]^{r-2} \cdot \cdot\left[P_{2,2}^{(r-1)}\right],
$$

where $P_{2,2}^{(k)}=0$ is satisfied by vertices $t, t^{\prime} k$ times removed from each other. If $F_{r, r}$ is given, the form $G$ and each of its factors is rationally known. Each of the forms $P^{(k)}$ is a rational covariant of $P^{(1)}$. For if we combine $P^{(1)}$ with $P^{(1)}$ (as $P^{(1)}$ was combined with $P^{(2)}$ in $\S 4$ to produce $P^{(3)}$ ) we obtain $P^{(2)}$; and in general $P^{(1)}$ combined with $P^{(k-1)}$ produces $P^{(k)}$. Moreover if $F_{r, r}$ has the closure property, $P_{2,2}^{(1)}$ has the closure property for the $n t^{\prime} \mathrm{s}$ arranged cyclically; and $P_{\mathbf{2}, 2}^{(k)}$ has the closure property for the $k$ th power of this cyclic arrangement.

(20) The form $P_{2,2}^{(1)}$ is the form $G$ associated with the planar Poncelet polygons of $n$ sides determined by a 2,2 form in $t, \sigma$.

For if $P_{2,2}^{(1)}$ has the closure property for $t=t_{1}, t_{2}, \cdots, t_{n}$ we mark on a norm-conic $C^{2}$ with parameter $t$ the closed sets of $n$ points $t_{1}, \cdots, t_{n}$ and determine thereby $\infty^{1}$ planar polygons. For given $t=t_{1}$, adjacent vertices are given by $t^{\prime}=t_{n}, t_{2}$ in $P_{2,2}^{(1)}=0$. The coördinates $\xi$ of a line which cuts $C^{2}$ in $t_{1}, t_{2}$ are proportional to $1, t_{1}+t_{2}, t_{1} t_{2}$. Since $P_{2,2}^{(1)}$ is quadratic in these coördinates for $t, t^{\prime}=t_{1}, t_{2}$, it is quadratic in $\xi$. Thus the sides $\xi$ of the $\infty^{1}$ polygons envelop a conic $K^{2}$. If $\sigma$ is any parameter on $K^{2}$ the incidence condition of $C^{2}, K^{2}$ is a $(2,2)$ form in $t, \sigma$ of such sort that $P_{2,2}^{(1)}=0$ is the condition that $t, t^{\prime}$ are on the same side $\sigma$.

The same process of composition of $P_{2,2}^{(j)}$ with $P_{2,2}^{(1)}$ enables us to get any two vertices $t^{\prime}$ at like distance from $t$. Thus if $n$ is odd, $n=2 \nu+1$, the sequence of distinct forms $P$ closes with

$$
P_{2,2}^{(1)}, \quad P_{2,2}^{(2)}, \cdots \cdot, P_{2,2}^{(\nu)} \quad(n=2 \nu+1) ;
$$

if however $n$ is even, $n=2 \nu$, the sequence of forms $P$ is

$$
P_{2,2}^{(1)}, \quad P_{2,2}^{(2)}, \cdot \cdot, P_{2,2}^{(\nu)} \quad(n=2 \nu),
$$

and $P_{2,2}^{(\nu)}$ is $\left(I_{1,1}\right)^{2}$, i. e., the parameters of opposite vertices of the polygon are in a quadratic involution. 
Similarly $F_{r, r}$ will determine a series of forms symmetric and of degrees 2,2 in $\tau, \tau^{\prime}$ comprising, for $n=2 \nu+1$,

and, for $n=2 \nu$,

$$
R_{2,2}^{(1)}, \quad R_{2,2}^{(2)}, \cdots \cdot, R_{2,2}^{(\nu)}
$$

$$
R_{2,2}^{(1)}, \quad R_{2,2}^{(2)}, \cdots, R_{2,2}^{(4)},
$$

where $R_{2,2}^{(\nu)}=\left(J_{1,1}\right)^{2}$,. i. e., the parameters of opposite faces of the polygon are in a quadratic involution.

Provisionally we refer to the $r$-ads of $t$ 's in the above scheme in the order given as $\tau_{1}, \cdots, \tau_{n}$ (later, for odd $r$ at least, a much better choice can be made). Then the scheme of $r$-ads of $\tau$ 's coördinated to $t_{1}, \cdots, t_{n}$ is

$$
\tau_{n-r+2} \cdots \tau_{n} \tau_{1}, \tau_{n-r+3} \cdots \tau_{n} \tau_{1} \tau_{2} \cdots \cdot \tau_{n-r+1} \tau_{n-r+2} \cdots \tau_{n}
$$

We consider now the resultant in $t$ of $P_{2,2}^{(1)}$ and $F_{r, r}$, a form of degree $2 r$ in $t^{\prime}$ and $\tau$. This resultant furnishes for $t^{\prime}=t_{1}$ the values $\tau=\tau_{n-r+3}, \cdots, \tau_{n}$ each twice, and the pairs of values $\tau_{n-r+2}, \tau_{1}$ and $\tau_{n-r+1}, \tau_{2}$ each once. Hence there can be rationally factored from this resultant a form $F_{r-2, r-2}$ which coördinates to $t_{1}$ all the faces $\tau$ on $t_{1}$ which are coördinated to $t_{1}$ by $F_{r, r}$ except the extreme faces $\tau_{n-r+2}, \tau_{1}$. Thus $F_{r, r}$ has the rational factors $F_{r-2, r-2}$ and the residual factor $F_{2,2}^{(1)}$. If $F_{r, r}$ has the closure property each of these factors has the closure property and the $(r-2)$-ads and duads of $\tau$ 's for these factors which are ascribed to $t_{1}, \cdots, t_{n}$ respectively are

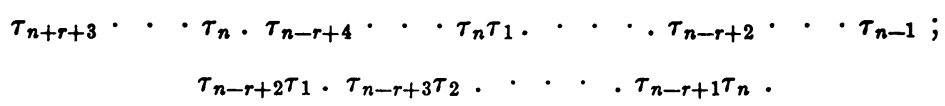

The factor $F_{r-2, r-2}$ is now poristic with polygons of $n$ vertices in $S_{r-2}$. We may think of the original polygon in $S_{r}$ as projected from a line upon an $S_{r-2}$. The $S_{r-2}$ 's, and the $S_{r-1}$ 's, or faces, of the original polygon are lost and the $S_{r-3}$ 's of the original polygon become the faces of the projected polygon.

Again we form the resultant of $P_{2,2}^{(1)}$ and $F_{r-2, r-2}$ and obtain the square of a rational factor $F_{r-4, r-4}$ which coördinates to $t^{\prime}=t_{1}$ the values $\tau_{n-r+4} \cdots$ $\tau_{n-1}$. Thus $F_{r-2, r-2}$ factors into $F_{r-4, r-4} F_{2,2}^{(2)}$, two poristic forms whose scheme of $\tau$ 's is

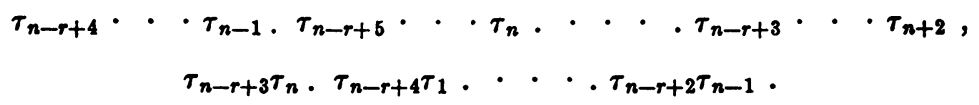

Continuing in this way to remove factors $F_{2,2}$ from $F_{r, r}$, which cuts off at each stage the extreme faces of the set on a given vertex, we finally wind up in one of two ways according as $r$ is odd or even. 
If $r=2 \rho$ we have the following complete resolution of $F_{r, r}$ :

$$
F_{r, r}=F_{2,2}^{(1)} \cdot F_{2,2}^{(2)} \ldots F_{2,2}^{(\rho)}
$$

with the following scheme for the successive poristic forms:

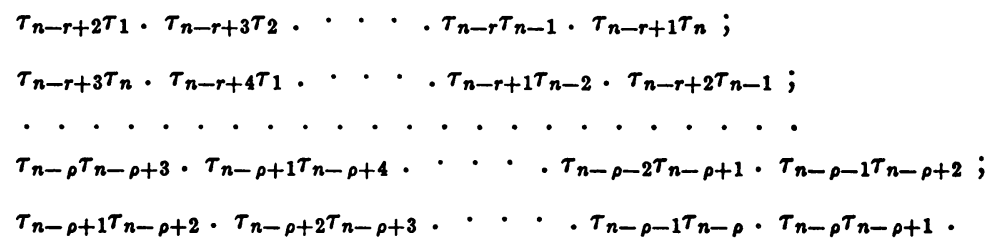

If $r=2 \rho+1$ we have the following complete resolution of $F_{r, r}$ :

$$
F_{r, r}=F_{2,2}^{(1)} \cdot F_{2,2}^{(2)} \cdot \cdot F_{2,2}^{(\rho)} \cdot F_{1,1} ;
$$

with the following scheme for the successive poristic forms:

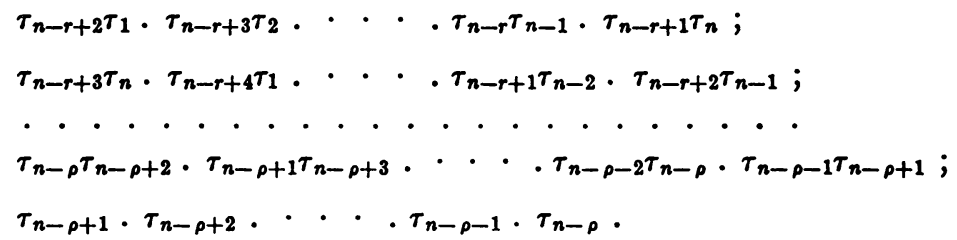

The $(2,2)$ forms $F_{2,2}$ in $(24)$ and $\left(24^{\prime}\right)$ are each poristic if $F_{r, r}$ is poristic. Each one therefore defines a system of planar Poncelet polygons. But the cyclic arrangement in these various planar systems is not the cyclic arrangement $C=(123 \cdots n)$ of the scheme (18). Indeed for the forms $F^{(1)}, \cdots$, $F^{(\rho)}$ in (24) the cyclic arrangement is $C^{2 \rho-1}, C^{2 \rho-3}, \ldots, C^{3}, C^{1}=C$ respectively; whereas for the forms $F^{(1)}, \cdots, F^{(\rho)}$ in $\left(24^{\prime}\right)$ the cyclic arrangement is $C^{2 p}, C^{2 p-2}, \cdots, C^{4}, C^{2}$.

Hence to construct a poristic form of the type (24) we begin with a form $F_{2,2}^{(p)}$ determined by planar Poncelet polygons on $n$ sides and construct its "iterations" $F_{2,2}^{(0-1)}, \ldots$. The rational process for this is as follows. We eliminate $\tau$ from $F_{2,2}^{(\rho)}(t)=0$ and $F_{2,2}^{(\rho)}\left(t^{\prime}\right)=0$ and, after separating the factor $\left(t-t^{\prime}\right)^{2}$ obtain $G_{2,2}^{(\rho)}\left(t, t^{\prime}\right)$, which coördinates adjacent vertices $t, t^{\prime}$. The resultant in $t$ of $G_{2,2}^{(\rho)}\left(t, t^{\prime}\right)$ and $F_{2,2}^{(\rho)}(t)$ is the product of $F_{2,2}^{(\rho)}$ and $F_{2,2}^{(\rho-1)}$, the original form and its iteration. The resultant in $t$ of $G_{2,2}^{(\rho)}\left(t, t^{\prime}\right)$ and $F_{2,2}^{(\rho-1)}$ is the product of $F_{2,2}^{(\rho)}$ and $F_{2,2}^{(\rho-2)}$, etc. Obviously the form $F_{r, r}$ constructed in (24) with these factors is poristic with the configurations required.

For $r=2 \rho+1$ there is, of the $r$ vertices on a face, one central vertex isolated, i.e., every $\tau$ determines one $t$ and vice versa. This projectivity between $\tau$ and $t$ is given by the form $F_{1,1}$ in $\left(24^{\prime}\right)$, and we shall now choose the 
order of the $n \tau$ 's so that $\tau_{i}$ corresponds to $t_{i}$ in this projectivity. Furthermore, we shall change the parameter $\tau$ in such a way that this projectivity is $t-\tau=0$, whence $\tau_{i}=t_{i}$. Then $\left(25^{\prime}\right)$ becomes

$$
\begin{aligned}
& \frac{t_{1}}{\tau_{n-\rho+1} \tau_{\rho+1}} \underset{\tau_{n-\rho+2} \tau_{\rho+2}}{\tau_{2}} \cdots \frac{t_{n-1}}{\tau_{n-\rho-1} \tau_{\rho-1}} \frac{t_{n}}{\tau_{n-\rho} \tau_{\rho}} \\
& \begin{array}{llllll}
\tau_{n-\rho+2} \tau_{\rho} & \tau_{n-\rho+3} \tau_{\rho+1} & \cdots & \tau_{n-\rho} \tau_{\rho-2} & \tau_{n-\rho+1} \tau_{\rho-1}
\end{array}
\end{aligned}
$$

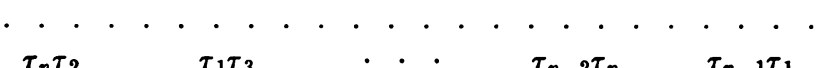

$$
\begin{aligned}
& \begin{array}{lllllll}
\tau_{1} & \tau_{2} & . & \tau_{n-1} & \tau_{n} & \left(\tau_{i}=t_{i}\right) .
\end{array}
\end{aligned}
$$

Due to the coalescence of $\tau^{\prime}$ 's with $t$ 's the form $F_{2,2}^{(\rho)}$ may now be regarded as a form which coördinates to $t_{1}$ the adjacent vertices $\tau_{n}, \tau_{2}=t_{n}, t_{2}$ of a planar polygon of $n$ sides. Hence if an $F_{2,2}(t, \tau)$ associated with planar Poncelet polygons of $n$ sides is given we construct the symmetric form $F_{2,2}^{(\rho)}\left(t, t^{\prime}\right)$ which coördinates adjacent vertices and construct the iterations $F_{2,2}^{(2-1)}\left(t, t^{\prime}\right), \cdots, F_{2,2}^{(1)}\left(t, t^{\prime}\right)$. If $t^{\prime}$ is replaced by $\tau$ in these forms and $F_{1,1}$ replaced by $t-\tau$, the form $F_{r, r}$ in $\left(24^{\prime}\right)$ is determined. Thus for $n=6, r=3$ we have the form (17) symmetric in $t, \tau$. The further condition on this form -invariance under the simultaneous change of sign of $t$ and $\tau$-is due to the fact that $n$ is even.

If now we interpret the form $F_{r, r}$ as the incidence condition of point $t$ of a rational norm curve $C^{r}$ in $S_{r}$ with $S_{r-1}$ of a rational norm curve $K^{r}$ in $S_{r}$, we may state briefly the following theorem for the generalized Poncelet polygons:

(26) For any value $n>2$ and $1<r<n$ there exist systems of $\infty^{1}$ Poncelet polygons $P_{n}$ in $S_{r}$ whose $n$ vertices run over a rational norm curve $C^{r}$ in $S_{r}$ while the $n$ faces envelop a rational norm curve $K^{r}$ in $S_{r}$. The corresponding algebraic forms $F_{r, r}(t, \tau)$ with the closure property for configurations $\Delta_{n, n}^{r, r}$ of the type (18) are for $r>2$ factorable as indicated in $(24),\left(24^{\prime}\right)$. The factors of $F_{r, r}$ are rational covariants constructed as described above of the form $F_{2,2}(t, \tau)$ associated with the planar Poncelet polygons of $n$ sides.

6. Poristic Forms $F_{3,3}$ With CONFigurations $\Delta_{n, n}^{3,3}(n<10)$

The poristic forms $F_{3,3}$ have a special interest in that the configurations which they admit can be visualized in ordinary space in association with the cubic curves $C^{3}, K^{3}$. If we look for possible poristic forms with configurations $\Delta_{n, n}^{3,3}$ our first task is the tabulation of arrangements of $n$ triads $\tau$ of three out of $n t$ 's such that each $t$ occurs in three triads. Here the concluding remark of $\$ 1$ and more especially the transitivity requirement [(9)\$1] are to be borne 
in mind. A first question which then naturally arises is the following: Can a pair of $t$ 's which occurs in one triad $\tau$ occur again in one or more other triads? If $t_{1}, t_{2}$ occur in triads $\tau_{1}, \tau_{2}$, the line joining the points $t_{1}, t_{2}$ of $C^{3}$ is on the planes $\tau_{1}, \tau_{2}$ of $K^{3}$. This line is then both a bisecant of $C^{3}$ and an axis of $K^{3}$, say a "secant-axis" of $C^{3}, K^{3}$. Since a line cannot be on more than two planes of $K^{3}$ we see that

No pair of t's can occur in more than two triads $\tau$.

Such pairs as occur in two triads will be called double pairs of the arrangement.

A next question is as to the number of double pairs in which a given $t$ may figure. Since the three planes of $K^{3}$ on a point $t$ of $C^{3}$ meet in three axes of $K^{3}$ on $t$, clearly a given $t$ cannot figure in more than three double pairs. If any double pairs occur, each $t$ must figure in the same number and therefore at least in one. Then the curves $C^{3}, K^{3}$ would have $\infty^{1}$ secant axes if the form $F_{3,3}$ is poristic, and on a point $t$ of $C^{3}$ there would be either 3 , or 2, or 1 of these secant axes. As Miss Cummings' examples show, any one of these three cases may arise. We shall consider then the relative situation of two cubic curves $C^{3}, K^{3}$ which have $\infty^{1}$ secant-axes and the further specialization which arises when the form $F_{3,3}$ is poristic.

(a) Through any point of $C^{3}$ there are three secant-axes of $C^{3}, K^{3}$. Then the three planes of $K^{3}$ on a point $t$ of $C^{3}$ meet in three secant-axes of $C^{3}, K^{3}$ on $t$ which meet $C^{3}$ again at $t_{1}, t_{2}, t_{3}$ respectively. Then the three planes of $K^{3}$ on $t_{1}$ are $t t_{1} t_{2}, t t_{1} t_{3}$, and $\pi$. The plane $\pi$ meets the other two planes in two bisecants of $C^{3}$ and these must be $t_{1} t_{2}$ and $t_{1} t_{3}$, else two bisecants of $C^{3}$ would meet in a point not on $C^{3}$, which is impossible if $C^{3}$ is a space cubic curve. Thus $\pi$ is the plane $t_{1}, t_{2}, t_{3}$ and the four faces of the tetrahedron $t, t_{1}, t_{2}, t_{3}$ are planes of $K^{3}$. Hence the symmetric relation $(\beta t)^{3}\left(\beta^{\prime} t^{\prime}\right)^{3}=0$ which expresses that $t t^{\prime}$ is a secant axis has one involutorial set $t, t_{1}, t_{2}, t_{3}$ and therefore has $\infty^{1}$ such sets which lie in an involution.

(28) If $C^{3}, K^{3}$ have $\infty^{1}$ secant-axes, three on any point of $C^{3}$, then there exist $\infty^{1}$ tetrahedra with vertices on $C^{3}$ and faces on $K^{3}$ and the incidence condition $F_{3,3}=0$ is necessarily poristic with configurations $\Delta_{4,4}^{3,3}$.

This case is referred to in (44) under the name of "Meyer's double quartettes."

(b) Through any point of $C^{3}$ there are two secant-axes of $C^{3}, K^{3}$. Then the three planes of $K^{3}$ on a point $t$ of $C^{3}$ meet in two secant-axes $t t_{1}, t t_{2}$ and in a third line unisecant to $C^{3}$. The plane $\tau$ of the two secant-axes is a plane of $K^{3}$ projectively related to point $t$ on the two secant-axes. If this projectivity 
is taken to be $t=\tau$ and if $(\beta t)^{2}\left(\beta^{\prime} t^{\prime}\right)^{2}=0$ is the symmetric relation on the parameters $t, t^{\prime}$ of points of $C^{3}$ on a secant axis, then the incidence condition of point $t$ and plane $\tau$ is $(t-\tau) \cdot(\beta t)^{2}\left(\beta^{\prime} \tau\right)^{2}=0$. If this $F_{3,3}$ is poristic then $(\beta t)^{2}\left(\beta^{\prime} \tau\right)^{2}=0$ admits closure of the planar Poncelet polygon type. Indeed in space the common secant-axes form the sides of closed polygons.

(29) If $C^{3}, K^{3}$ have $\infty^{1}$ secant-axes, two on any point of $C^{3}$, then there are two on any plane of $K^{3}$. The two curves $C^{3}, K^{3}$ are perspective, i.e., plane $\tau$ is or. like-named point $t$ and the incidence condition is $(t-\tau) \cdot(a \tau)^{2}(\alpha t)^{2}=0$ where $(a \tau)^{2}(\alpha t)^{2}$ is symmetric in $t, \tau$. If further the $F_{3,3}$ form is poristic then $C^{3}, K^{3}$ admit Poncelet polygons of the type $[\$ 5, r=3]$.

(c) Through any point of $C^{3}$ there is one secant-axis of $C^{3}, K^{3}$. The three planes of $K^{3}$ on $t$ meet in three lines one of which is a secant-axis and the other two are unisecant to $C^{3}$. Thus a plane $\tau$ of $K^{3}$ contains one secant axis and meets $C^{3}$ in a further point $t$. Thus again $C^{3}$ and $K^{3}$ are perspective with a plane $\tau$ cutting out a secant-axis and $t=\tau$; and a point $t$ lying on a secantaxis and a plane $\tau=t$. Points $t, t^{\prime}$ of a secant-axis satisfy a symmetric form $(\beta t)\left(\beta^{\prime} t^{\prime}\right)=0$, i.e. lie in an $I_{1,1}$; whence the secant-axes are generators $\lambda$ of a quadric $Q$ containing the points of $C^{3}$ and the planes of $K^{3}$. The incidence condition of point $t$ and plane $\tau$ is $(t-\tau) \cdot(a \tau)^{2}(\alpha t)^{2}=0$ where $(a \tau)^{2}(\alpha t)^{2}=0$ is imprimitive since each $t$ of a pair on a secant-axis determines both $\tau$ 's of a pair on that secant-axis and conversely.

(30) If $C^{3}, K^{3}$ have $\infty^{1}$ secant-axes, one on any point of $C^{3}$, then also there is one on every plane of $K^{3}$. The curves $C^{3}, K^{3}$ are perspective and lie on the same quadric. The factor of the incidence condition residual to $t-\tau$ is imprimitive and coördinates the pairs $t, t^{\prime}$ and $\tau, \tau^{\prime}$ on the same secant-axis. If further the $F_{3,3}$ form is poristic then $C^{3}, K^{3}$ admit the cyclically imprimitive configurations of the type $[\S 3,(13)]$.

Since (28), (29), (30) exhaust the cases of arrangements with double pairs we have the following theorem :

(31) All poristic forms $F_{3,3}$ with double pairs are included in the general aggregates of $\$ 3$ and $\$ 5$.

Since for a given value of $n$ the possible arrangements which are considered in $\$ 3$ and $\$ 5$ are easily tabulated we shall consider only configurations without double pairs. This materially reduces the number of possible configurations. For example, it is easily shown for $n=7$ that

(32) The only poristic form $F_{3,3}$ with configurations $\Delta_{7,7}^{3,3}$ which have no double pairs is that of White. 
We examine now the possible configuration $\Delta_{8,8}^{3,3}$ without double pairs. Of the 28 possible pairs formed from $8 t^{\prime}$ 's, 24 must appear in the 8 triads $\tau$, and 4 pairs must be missing. Because of the transitivity each $t$ must appear in one of the missing pairs. We take these missing pairs to be 12, 34, 56, 78. Since three of the triads must contain 1 and three others must contain 2, there remain two triads which contain neither 1 nor 2 . These must each contain one figure out of each of the three other pairs and may be chosen to be 357.468. In one triad the pair 13 occurs and this triad cannot contain 5 or 7 since the pairs 35,37 already occur. The triad may contain 6 or 8 and since 68 occurs as a pair in the first two triads the choice of 6 or 8 is not material. Hence we choose a triad 136. The triad containing the pair 15 can contain also 4 or 8 . Again the choice is not material and we select 158 and must then also select 147. The triad containing 23 must contain 8 since the pair 34 is not to occur and the pairs $35,36,37$ already occur. Similarly we find, for the three triads containing $2,238,245,267$. Thus the only type of $\Delta_{8,8}^{3,3}$ without double pairs is

$$
357.468 .136 .158 .147 .238 .245 .267 \text {. }
$$

We shall call these triads in the order written

$$
\tau=a, b, g, c, e, f, h, d,
$$

so that we have isolated pairs of $\tau$ 's ( $\tau$ triads without a common $t$ ) $a b, c d, e f, g h$ which correspond in order to the pairs of $t$ 's $12,34,56,78$ in the sense that the pair of $\tau$ 's , $a b$, without a common $t$ contain all the $t$ 's except the pair 12.

The group of the array (33) is of order 48 and abstractly identical with the product of a $g_{4 !}$ (permutations of the four pairs) and an interchangeable $g_{2}$ defined by the element (12)(34)(56)(78). For the array is unaltered by (16472538), a cyclic element of period 4 on the four pairs ; and by (357)(468), a cyclic element of period 3 on the four pairs. These generate $g_{41}$. If every pair is unaltered and 1 is unaltered, then 2 is also unaltered. If now 3 in 136 interchanges with 4 in 147 then 6 must go into 7 and the pairs are altered. Hence (12)(34)(56)(78) is the only element other than the identity which leaves each pair unaltered and it is obviously invariant in the $g_{48}$.

Let us assume that there exists a poristic form $F_{3,3}$ with configurations $\Delta_{8,8}^{3,3}$ of the type (33). Then the form $G_{6,6}$ symmetric in $t, t^{\prime}$, which are in a common triad, coördinates to $t=t_{1}$ the values $t^{\prime}=t_{3}, t_{4}, t_{5}, t_{6}, t_{7}, t_{8}$. The resultant in $t$ of $G_{6,6}\left(t, t^{\prime}\right)$ and $F_{3,3}(t, \tau)$ coördinates to $t^{\prime}=t_{1}$ the pair $\tau=a, b$ three times and the other three pairs each twice, whence this resultant has a rational factor $F_{2,2}\left(t^{\prime}, \tau\right)$ which coördinates to $t^{\prime}=t_{1}$ or $t^{\prime}=t_{2}$ the same pair 
$\tau=a, b$ and vice versa. The form $G_{2,2}$ in $t, t^{\prime}$ formed for $F_{2,2}(t, \tau)$ becomes the square of a symmetric form $F_{1,1}\left(t, t^{\prime}\right)$, whence the pairs $12,34,56,78$ are pairs of a quadratic involution and by a similar argument the pairs $a b, \cdots$, $g h$ are in involution. If we take the double points of these involutions to be $0, \infty$ then the four pairs of $t$ 's are determined by the equations

$$
t^{2}=l^{2}, m^{2}, n^{2}, r^{2}
$$

and the four pairs of $\tau$ 's by the equations

$$
\tau^{2}=\lambda^{2}, \mu^{2}, \nu^{2}, \rho^{2} .
$$

Moreover, in $F_{2,2}$ the same pair of $\tau$ 's is determined by either $t$ of a pair, whence $F_{2,2}$ is bilinear in $t^{2}$ and $\tau^{2}$, or the values $l^{2}, m^{2}, n^{2}, r^{2}$ and $\lambda^{2}, \mu^{2}, \nu^{2}, \rho^{2}$ have the same double ratio.

The resulting canonical form of $\Delta_{8,8}^{3,3}$ is

$$
\begin{gathered}
t_{1}, \cdots, t_{8}=l,-l, m,-m, n,-n, r,-r ; \\
\tau_{\alpha}, \cdot \cdots, \tau_{h}=\lambda,-\lambda, \mu,-\mu, \nu,-\nu, \rho,-\rho .
\end{gathered}
$$

If now we can find a form $F_{3,3}$ which admits this one configuration then $F_{3,3}$ is necessarily poristic. For it is easily verified from (34) and (33) that the form $F_{3,3}{ }^{\prime}$ obtained by changing the sign of $\tau$ in $F_{3,3}$ together with the form $F_{2,2}$ above combine to make an $F_{5,5}$ which admits the complementary configuration. If we examine the incidences for $F_{3,3}$ we see that $F_{3,3}$ must be unaltered when $t, \tau$ both change sign. Hence $F_{3,3}$ contains only 8 terms and 12 of the incidences imply the remaining 12 . These 12 conditions on the 7 ratios of the coefficients of $F_{3,3}$ and the 8 constants $\lambda, \mu, \nu, \rho, l, m, n, r$ leave at least three degrees of freedom. But $\lambda, l$ may each be taken as 1 by proper choice of the unit point for $t, \tau$, so that there remains at least one degree of freedom for the form and its variable configuration. An attempt at this point, where the relative simplicity of the configuration (34) seemed favorable, to impose the algebraic conditions led to relations which were unmanageable and further geometric light on the required form $F_{3,3}$ seemed necessary.

Consider then the possible ways in which the $\Delta_{8,8}^{3,3}$ can degenerate by coincidences of the $t^{\prime}$ s or $\tau$ 's. Two planes $\tau$ can coincide only if some of the $t$ 's coincide. If two planes coincide they may be either (a) two planes of a pair such as $a=357, b=468$; or (b) two planes from different pairs as $g=136$, $c=158$. When $a$ coincides with $b$ the points $3,5,7$ coincide with $4,6,8$ and three cases arise according as there are three, one, or no coincidences in the pairs $34,56,78$. Thus we have $\left(a_{1}\right) 3=4,5=6,7=8 ;\left(a_{2}\right) 3=4,5=8,6=7$; $\left(a_{3}\right) 3=6,5=8,7=4$. The case $\left(a_{1}\right)$ is impossible since the three planes 
$a=357, c=157, d=257$ of $K^{3}$ are on a bisecant 57 of $C^{3}$. Also the case $\left(a_{3}\right)$ cannot occur since 3,4 is one pair and 5,6 another pair of a quadratic involution, whence if 3 coincides with 6,4 must coincide with 5 . Thus in case $\left(a_{3}\right)$ the points $3,4,5,6,7,8$ all would coincide. The case $\left(a_{2}\right)$ leads to the coincidences $a=b, e=g, f=h$ among the planes, i.e., two planes of a pair and two other pairs coincide as with the points. The case $\left(a_{2}\right)$ can happen just twice since the points of a pair can coincide only at $t=0$ and $t=\infty$. In case (b), $g=136 \equiv C=158$, either $\left(b_{1}\right) 3=5,6=8$, or $\left(b_{2}\right) 3=8,5=6$. In the case $\left(b_{2}\right)$ since $3=8$ then $4=7$ and with $5=6$ we have again case $\left(a_{2}\right)$. In the case $\left(b_{1}\right)$ we must have also $4=6,5=7$, i.e. $3=5=7$ and $4=6=8$, i.e. three pairs coincide without a coincidence among the members of a pair. The configuration of planes behaves similarly since now $c=e=g, d=f=h$. Moreover, this case $\left(b_{1}\right)$ can happen just twice. For in a pencil of octavics there are 14 coincidences among the roots, a $k$-fold root counting as $k-1$ coincidences. Thus the two instances of case $\left(a_{2}\right)$ contribute 6 coincidences and each case $\left(b_{1}\right)$ contributes 4 coincidences. With the help of these degenerate configurations we shall prove the following theorem :

(35) There exists a poristic form $F_{3,3}$ containing one absolute constant which admits $\infty^{1}$ configurations $\Delta_{8,8}^{3,3}$ of the type (33). The eight points and eight planes on each configuration are distinct except in two cases each of types $\left(\mathrm{a}_{2}\right)$ and $\left(\mathrm{b}_{1}\right)$ above. This is the only poristic $F_{3,3}$ with double octettes other than that of the eight-sided Poncelet polygon in space and that of the cyclically imprimitive type which arises from a division of eight things into four sets of two.

The equation $F_{3,3}=0$ is to be unaltered by the simultaneous change of sign of $t$ and $\tau$. It contains therefore terms $t^{i} \tau^{j}$ such that either $i+j$ is even or $i+j$ is odd. Either case may be reduced to the other by the substitution $\tau^{\prime}=1 / \tau$ which leaves the involution $\tau^{\prime}=-\tau$ unaltered. We shall then take $F_{3,3}$ to be

$$
\lambda_{1} \tau^{3} t^{3}+\lambda_{2} \tau^{3} t+\lambda_{3} \tau^{2} t^{2}+\lambda_{4} \tau^{2}+\lambda_{5} \tau t^{3}+\lambda_{6} \tau t+\lambda_{7} t^{2}+\lambda_{8} .
$$

If now in a degenerate configuration of type $\left(a_{2}\right)$ two points of a pair come together at $t=\infty$, then two planes of a pair come together at $\tau=0$. The two coincident pairs of points will be taken as $t= \pm 1$; of planes as $\tau= \pm 1$, whence all remaining constants are arbitrary. Then the scheme of parameter values of the eight $t$ 's and $\tau$ 's for this degenerate configuration, when written as in (34), becomes

$$
\begin{gathered}
\infty, \infty, 1,-1,1,-1, b,-b, \\
\beta,-\beta, 1,-1,-1,1,0,0 .
\end{gathered}
$$


The incidences implied by this scheme furnish just seven linear equations for the determination of $\lambda_{1}, \cdots, \lambda_{8}$ and the resulting values are

$$
\begin{aligned}
\lambda_{1}=-\lambda_{5} & =(1-3 \beta), \\
\lambda_{2} & =2 b^{2}(\beta-1)+(\beta+1), \\
\lambda_{3} & =(\beta+1)[b(\beta-2)+2(\beta-1)], \\
\lambda_{4} & =-(\beta+1)[2 b(\beta-1)+(\beta-2) b], \\
\lambda_{6} & =2 b^{2} \beta(\beta-1)+(1+\beta)(1-2 \beta), \\
-\lambda_{8}=\lambda_{7} & =b \beta(\beta+1) .
\end{aligned}
$$

The $F_{2,2}$ form bilinear in $t^{2}, \tau^{2}$ must be satisfied by the pairs $t^{2}, \tau^{2}=\infty, \beta^{2}$; 1,$1 ; b^{2}, 0$, whence it is

$$
\tau^{2}=\frac{\beta^{2}\left(t^{2}-b^{2}\right)}{t^{2}-\left(b^{2} \beta^{2}-\beta^{2}+1\right)} .
$$

For the second degenerate configuration of type $\left(a_{2}\right)$ the parameter scheme is

$$
\begin{gathered}
0,0, c,-c, c,-c, d,-d, \\
\delta,-\delta, \gamma,-\gamma,-\gamma, \gamma, \infty, \infty .
\end{gathered}
$$

For $\tau=\infty$ and $t=0$ the values of $c^{2}$ and $\gamma^{2}$ are found to be, in terms of $b$ and $\beta$, the following:

$$
c^{2}=-\lambda_{2} / \lambda_{1}, \quad \gamma^{2}=\lambda_{7} / \lambda_{4} .
$$

Also the $F_{2,2}$ form in $t^{2}, \tau^{2}$ must be satisfied by the pairs $t^{2}, \tau^{2}=0, \delta^{2} ; d^{2}, \infty$; $c^{2}, \gamma^{2}$. From the first two pairs we find

$$
\delta^{2}=b^{2} \beta^{2} / d^{2}, \quad d^{2}=b^{2} \beta^{2}-\beta^{2}+1 .
$$

The third pair $c^{2}, \gamma^{2}$, already known in terms of $b, \beta$, leads to the following condition on $b, \beta$ and therefore leaves only one constant :

$$
b=(1-2 \beta) / \beta .
$$

We now express all the quantities involved in terms of $\beta$ as follows :

$$
\begin{aligned}
\lambda_{1}=-\lambda_{5} & =\beta^{2}(1-3 \beta), & b & =(1-2 \beta) / \beta, \\
\lambda_{2} & =-d^{2}(1-3 \beta), & d^{2} & =3 \beta^{2}-4 \beta+2, \\
\lambda_{3} & =\beta(\beta+1)(3 \beta-2), & \delta^{2} & =(1-2 \beta)^{2} / d^{2}, \\
\lambda_{1} & =d^{2}(\beta+1)(1-2 \beta), & c^{2} & =d^{2} / \beta^{2}, \\
\lambda_{6} & =\beta(1-3 \beta)(1-2 \beta)(\beta-2), & & \gamma^{2}=\beta^{2} / d^{2} . \\
-\lambda_{8}=\lambda_{7} & =\beta^{2}(\beta+1)(1-2 \beta) ; & &
\end{aligned}
$$


The $F_{2,2}$ form in $t^{2}, \tau^{2}$ now leads to

$$
\tau^{2}=\frac{\beta^{2}\left(t^{2}-b^{2}\right)}{t^{2}-d^{2}} .
$$

It is necessary to verify also the further incidences for the configuration $\left(34^{\prime \prime}\right)$. When $t=c, \tau=\infty, \delta, \gamma$. The quadratic in $\tau$ with roots $\delta, \gamma$ obtained from (36), furnishes values

$$
\begin{aligned}
\delta+\gamma & =c \frac{\lambda_{1} c^{2}-\lambda_{6}}{\lambda_{3} c^{2}+\lambda_{4}}=c \frac{\beta(3 \beta-1)}{d^{2}}, \\
\delta \gamma & =\frac{\lambda_{7}\left(c^{2}-1\right)}{\lambda_{3} c^{2}+\lambda_{4}}=\frac{\beta(2 \beta-1)}{d^{2}} .
\end{aligned}
$$

On squaring $\delta+\gamma$ and $\delta \gamma$ we find that these values are consistent with the relations (37). When $t=d, \tau=\delta,-\gamma,-\gamma$, from which we have in (36)

$$
\delta-2 \gamma=1 / d, \quad \gamma^{2}-2 \gamma \delta=-\beta(3 \beta-2) / d^{2}, \quad \gamma^{2} \delta=\beta^{2}(1-2 \beta) / d^{3} .
$$

Again these values prove to be consistent with the relations (37). There remains the determination of the signs of $c, \gamma, d, \delta$. Let

$$
d=\sqrt{3 \beta^{2}-4 \beta+2} \text {. }
$$

Then from (37) $\delta=\epsilon_{1}(1-2 \beta) / d, c=\epsilon_{2} d / \beta, \gamma=\epsilon_{3} \beta / d$, where $\epsilon_{1}, \epsilon_{2}, \epsilon_{3}$ are +1 or -1 . From the values of $\delta+\gamma, \delta \gamma$, and $\delta-2 \gamma$ just above, we find that

$$
\delta=(1-2 \beta) / d, \quad c=-d / \beta, \quad \gamma=-\beta / d .
$$

Up to this point we have proved that

(41) The form $F_{3,3}$ in (36) with coefficients $\lambda_{1}, \cdots, \lambda_{8}$ expressed in (37) as polynomials in the arbitrary constant $\beta$ admits the two degenerate configurations $\left(34^{\prime}\right)$ and (34') with values $c, \gamma, \delta, d, b$ given in (40), (39), and (37).

We still have to prove that this form $F_{3,3}$ is poristic. A study of the general configuration in (34) shows that the $(5,5)$ form with configurations $\Delta_{\mathbf{8 , 8}}^{\mathbf{5 , 5}}$ which is complementary to $F_{3,3}$ with configurations $\Delta_{8,8}^{3,3}$ breaks up into a product of the form $F_{2,2}$ and a form $\bar{F}_{3,3}$ which arises from $F_{3,3}$ by a change of sign of $\tau$ alone. If now the product $F_{3,3} \cdot \bar{F}_{3,3} \cdot F_{2,2}$ can be expressed as a determinant $D_{8,8}$ as in (5) which is necessarily poristic then each factor such as $F_{3,3}$ is necessarily poristic. The form $F_{2,2}$ is obtained from (38),

Also

$$
F_{2,2} \equiv \tau^{2} t^{2}-d^{2} \tau^{2}-\beta^{2} t^{2}+d^{2} \delta^{2}
$$

$$
F_{3,3} \cdot \bar{F}_{3,3} \equiv\left[\lambda_{3} \tau^{2} t^{2}+\lambda_{4} \tau^{2}+\lambda_{7}\left(t^{2}-1\right)\right]^{2}-\tau^{2} t^{2}\left[\lambda_{1} t^{2}\left(\tau^{2}-1\right)+\lambda_{2} \tau^{2}+\lambda_{6}\right]^{2} .
$$


For the determinant $D_{8,8}$ we know that the two octavics in $t$ determined by the configurations $\left(34^{\prime}\right)$ and $\left(34^{\prime \prime}\right)$ correspond respectively to the two octavics in $\tau$ similarly determined, so that a provisional form of $D_{8,8}$ is

$$
D_{8,8}=\left|\begin{array}{lc}
\left(t^{2}-1\right)^{2}\left[\beta^{2} t^{2}-(1-2 \beta)^{2}\right] & t^{2}\left(t^{2}-c^{2}\right)^{2}\left(t^{2}-d^{2}\right) \\
\mu^{2} \tau^{2}\left(\tau^{2}-\beta^{2}\right)\left(\tau^{2}-1\right)^{2} & \left(\tau^{2}-\gamma^{2}\right)^{2}\left(\tau^{2}-\delta^{2}\right)
\end{array}\right|
$$

where $\mu$ is a constant to be determined. We have to show that $\mu, \nu$ can be determined so that

$$
D_{8,8}=\nu F_{2,2} \cdot F_{3,3} \cdot \bar{F}_{3,3} .
$$

A tedious direct calculation can be avoided here by the following argument. If we apply (38) regarded as a substitution on $\tau^{2}$ to $D_{8,8}$ the second row becomes a numerical multiple of the first row provided that

$$
\mu=\frac{\beta^{4}(1-3 \beta)^{2}}{d^{6}(\beta+1)^{2}} .
$$

With this value of $\mu, D_{8,8}$ must contain the factor $F_{2,2}$. The residual factor of $D_{8,8}$ is a $(3,3)$ form $G_{3,3}(s, \sigma)$ in $s=t^{2}$ and $\sigma=\tau^{2}$ which by inspection is seen to be satisfied by the following pairs of values of $s$ and $\sigma$ :

$$
\begin{array}{llll}
s=\infty, & \sigma=1,1,0 ; & s=0, & \sigma=\gamma^{2}, \gamma^{2}, \infty ; \\
s=1, & \sigma=1,0, \beta^{2} ; & s=c^{2}, & \sigma=\delta^{2}, \gamma^{2}, \infty ; \\
s=b^{2}, & \sigma=\beta^{2}, 1,1 ; & s=d^{2}, & \sigma=\delta^{2}, \gamma^{2}, \gamma^{2} .
\end{array}
$$

These values are sufficient to identify $G_{3,3}(s, \sigma)$ as a poristic form with configurations $\Delta_{4,4}^{3,3}$ (the two given here being degenerate) which arises geometrically from tetrahedra inscribed in one cubic curve and circumscribed to another. But the product $F_{3,3} \cdot \bar{F}_{3,3}$ is satisfied by the same values and therefore is a multiple of the residual factor $G_{3,3}(s, \sigma)$ of $D_{8,8}$. Hence $F_{3,3}$ is a poristic factor of $D_{8,8}$.

This completes the proof of the theorem (35) except for the verification of the existence of the two degenerate configurations of type $\left(b_{1}\right)$. The two octavics in $t$ found in $D_{8,8}$ have a jacobian of order 14 which must have the factors $t, t^{2}-1, t^{2}-c^{2}$, indicating the coincidences $0, \infty, \pm 1, \pm c$ found in $\left(34^{\prime}\right)$ and $\left(34^{\prime \prime}\right)$. The remaining octavic factor of this jacobian should be a perfect square indicating the four coincidences in triples found in the two configurations of type $\left(b_{1}\right)$. If we take these two octavics in the form

$$
\left(t^{2}-1\right)^{2}\left(t^{2}-b^{2}\right), \quad t^{2}\left(t^{2}-c^{2}\right)\left(t^{2}-d^{2}\right)
$$

and pass to the parameter $b$ by using $\beta=1 /(b+2)$, in which case $c^{2}=2 b^{2}$ $+4 b+3, d^{2}=c^{2} /(b+2)^{2}$, the octavic factor of the jacobian turns out to be

$$
\left[t^{4}+2 b t^{2}-b d^{2}(b+2)\right]^{2} \text {. }
$$


We shall close with a demonstration that there are no "double nonettes" or configurations $\Delta_{9,9}^{3,3}$ for a form $F_{3,3}$ which are of particular interest. The cases obviously present according to the above discussion are first of all the Poncelet polygons of 9 sides in space. Secondly, for the cyclically imprimitive cases the $9 t_{i}=\tau_{i}(i=1, \cdots, 9)$ must be arranged in three rows of three, and to a $t$ in one row there must correspond the three $\tau$ 's in the next row. This case is imprimitive and arises from a periodic collineation of period three by transformation of the third order.

There remain the cases in which the 9 triads of $t$ 's contain no repeated pair of $t$ 's. The triads then contain 27 pairs of $t$ 's and 9 pairs of $t$ 's are not found in the triads. Because of the transitivity of the triads each $t$ must occur in two of the 9 pairs, and these may therefore be written in the cyclic order

$$
12.23 .34 .45 .56 .67 .78 .89 .91 \text {. }
$$

'The group which permutes the triads must permute these isolated pairs and therefore is either the dihedral $g_{2.9}$ constructed for the cycle 123456789 or a transitive subgroup of it. The only transitive subgroup of $g_{2.9}$ is the cyclic $g_{9}$ and the triads must therefore admit this cyclic group.

The formation of the triads is now the problem of arranging the 27 diagonals of a polygon of 9 vertices $1,2, \cdots, 9$ into 9 triangles three of which have a vertex at each vertex of the polygon. Moreover, the 9 triangles must permute cyclically under the cyclic $g_{9}$. Since 1 occurs with 9 and 2 in the omitted pairs it must occur with each of the other $t$ 's in some triad. There is therefore a triad $13 x_{1}$, and on applying $g_{9}$ we have triads $13 x_{1}, 24 x_{2}, 35 x_{3}$. The 9 triads cannot permute cyclically in three sets of three, since $35 x_{3}$ permutes into $46 x_{4}$ which cannot be $13 x_{1}$. Hence they permute in a cyclic set of 9 which is

$$
13 x_{1} \cdot 24 x_{2} \cdot 35 x_{3} \cdot 46 x_{4} \cdot 57 x_{5} \cdot 68 x_{6} \cdot 79 x_{7} \cdot 81 x_{8} \cdot 92 x_{9} .
$$

Since 1 and 3 already occur with 2, 4, 9 in the omitted pairs and with 5 and 8 in the triads the first triad must be 136 or 137 . The two triangles hereby obtained have sides which in order skip 1, 2, 3 vertices or 1, 3, 2 vertices of the original polygon. Thus the two cases are not distinct, and we choose

$$
136.247 .358 .469 .571 .682 .793 .814 .925 \text {, }
$$

observing that the choice of 6 rather than 7 cuts the group of the triads from the dihedral $g_{2.9}$ to a cyclic $g_{9}$.

We denote the triads in order by $\tau=a, b, \cdots, g, h, i$, and assume that a poristic form $F_{3,3}$ exists which admits $\infty^{1}$ configurations of the type (42). The symmetric form $G_{6,6}$ in $t, t^{\prime}$, where $t, t^{\prime}$ is a pair in a $\tau$ triad, coördinates 
to $t$ all the vertices of the polygon except $t$ itself and the two adjacent vertices. The resultant of $G$ and $F_{3,3}$ coördinates to $t=t_{1}$ the values $\tau=c$ three times, $\tau=i$ once and $\tau=a, b, d, e, f, g, h$ each twice. Hence there is a form $F_{1,1}$ which coordinates $t_{1}$ and $i$; a form $F_{1,1}$ which coördinates $t_{1}$ and $c$; and a form which contains the factor $F_{3,3}$ and a residual factor $F_{4,4}$ which coördinates to $t_{1}$ the values $\tau=b, d, f, g$,

The significance of these factors is clear from the figure of the polygon and the 9 triangles. On fixing $\tau=a=136$ we have a triangle whose sides $13,36,61$ skip respectively the vertices $2 ; 4,5 ; 7,8,9$. Thus $\tau=a$ uniquely determines $t=t_{2}$ as in $F_{1,1}$; also it uniquely determines $t=t_{8}$, the middle vertex of $7,8,9$ as in $F_{1,1}{ }^{\prime}$; it also uniquely determines the adjacent pair 7, 9 by a form $F_{2,2}$; as well as the pair 4,5 by a form $F_{2,2}^{\prime}$. Hence $F_{4,4}$ factors into $F_{2,2} \cdot F_{2,2}{ }^{\prime}$. We write for convenience the table of values of these four forms, each poristic if $F_{3,3}$ is poristic:

$\begin{array}{llllllllll}F_{2,2}: & \frac{a}{7,9} & \frac{b}{8,1} & \frac{c}{9,2} & \frac{d}{1,3} & \frac{e}{2,4} & \frac{f}{3,5} & \frac{g}{4,6} & \frac{h}{5,7} & \frac{i}{6,8} \\ F_{2,2}^{\prime}: & 4,5 & 5,6 & 6,7 & 7,8 & 8,9 & 9,1 & 1,2 & 2,3 & 3,4 \\ F_{1,1}: & 2 & 3 & 4 & 5 & 6 & 7 & 8 & 9 & 1 \\ F_{1,1}^{\prime}: & 8 & 9 & 1 & 2 & 3 & 4 & 5 & 6 & 7\end{array}$

In $F_{2,2}, \tau=a$ determines $t=7,9$; these determine in $F_{2,2}{ }^{\prime}, \tau=c, d, e, f$; and these determine in $F_{2,2}, t=2,3$ each twice and $t=1,4,5,9$ each once. Hence there is a 2,2 form which coördinates to $\tau=a$ the values $t=2,3$ and this factors into $F_{1,1} \cdot F_{1,1}^{\prime \prime}$, where $F_{1,1}^{\prime \prime}$ coördinates $\tau=a$ to $t=3$. Then $t=2$ in $F_{1,1}$ determines $\tau=a$ and this in $F_{1,1}^{\prime \prime}$ determines $t^{\prime}=3$, whence there is a cyclic collineation which permutes $t=t_{1}, \cdots, t=t_{9}$ cyclically. If we take the fixed points of this collineation to be $0, \infty$, the parameters of the $9 t$ 's are

$$
k, k \epsilon, k \epsilon^{2}, \cdots, k \epsilon^{8} \quad \text { ( } \epsilon \text { a primitive root of unity). }
$$

If we take the parameter system for $\tau$ in such wise that the form $F_{1,1}$ is $\tau-t$ then the parameters of the $9 \tau$ 's in order are

$$
k \epsilon, k \epsilon^{2}, k \epsilon^{3}, \cdots, k \epsilon^{8}, k \text {; }
$$

and the form $F_{3,3}$ is merely

$$
F_{3,3}=\left(t-\epsilon^{8} \tau\right) \cdot(t-\epsilon \tau) \cdot\left(t-\epsilon^{4} \tau\right) .
$$

Such a product of positive powers of a cyclic collineation is obviously poristic.

This completes the survey of possible configurations $\Delta_{n, n}^{3,3}$ up to $n=10$ and leads to the following noteworthy theorem: 
(44) Of all possible poristic forms $F_{3,3}$ with configurations $\Delta_{n, n}^{3,3}(n<10)$ there are but three which do not degenerate into products of poristic forms of lower orders. The attached configurations are the double quartettes of Meyer, the double septettes of White, and the double octettes described in (35).

For $n=10$ the group problem of tabulating possible poristic arrangements is itself not a simple one.

UNIVERSTTY OF ILINOIS,

Urbana, ILL. 